Poznań Studies in Contemporary Linguistics 46(4), 2010, pp. 519-532

(C) School of English, Adam Mickiewicz University, Poznań, Poland

doi:10.2478/v10010-010-0026-2

\title{
A LOGIC-BASED APPROACH TO PROBLEMS IN PRAGMATICS
}

\author{
PIETER A.M. SEUREN \\ Max Planck Institute for Psycholinguistics, Nijmegen \\ pieter.seuren@mpi.nl
}

\begin{abstract}
After an exposé of the programme involved, it is shown that the Gricean maxims fail to do their job in so far as they are meant to account for the well-known problem of natural intuitions of logical entailment that deviate from standard modern logic. It is argued that there is no reason why natural logical and ontological intuitions should conform to standard logic, because standard logic is based on mathematics while natural logical and ontological intuitions derive from a cognitive system in people's minds (supported by their brain structures). A proposal is then put forward to try a totally different strategy, via (a) a grammatical reduction of surface sentences to their logico-semantic form and (b) via logic itself, in particular the notion of natural logic, based on a natural ontology and a natural set theory. Since any logical system is fully defined by (a) its ontology and its overarching notions and axioms regarding truth, (b) the meanings of its operators, and (c) the ranges of its variables, logical systems can be devised that deviate from modern logic in any or all of the above respects, as long as they remain consistent. This allows one, as an empirical enterprise, to devise a natural logic, which is as sound as standard logic but corresponds better with natural intuitions. It is hypothesised that at least two varieties of natural logic must be assumed in order to account for natural logical and ontological intuitions, since culture and scholastic education have elevated modern societies to a higher level of functionality and refinement. These two systems correspond, with corrections and additions, to Hamilton's 19thcentury logic and to the classic Square of Opposition, respectively. Finally, an evaluation is presented, comparing the empirical success rates of the systems envisaged.
\end{abstract}

KEYWORDS: Empirical adequacy of logic; Gricean maxims; natural logic; reflexivity the linguistic worldview; facets; cognitive domains; the domain matrix; on-line meaning.

\section{Introduction ${ }^{*}$}

The general hypothesis is that natural speakers start off with a basic-natural logic, based on natural cognitive functions, including the basic-natural way of dealing with

\footnotetext{
* I am grateful to one referee, whose highly apposite critical remarks and suggestions have been incorporated into the final text, for which, of course, I remain fully responsible.
} 
plural objects. This basic-natural logic is generally characterised by the fact (a) that logics are applications of set theory and that basic-natural set theory is subject to constraints not valid for standard set theory (Seuren 2010, Ch. 3), and (b) that the notions of truth and entailment are still, uncritically, dependent on (thinker's or) speaker's knowledge state $\mathbf{K}$ of the moment. To the extent that individuals (and societies) learn to take distance from any $\mathbf{K}$ of the moment, the logic gets more refined. ${ }^{1}$ As culture spreads, stricter thinking, resulting from functional pressure, leads to better generalizations and greater mathematical correctness, yielding ever more refined systems until the apogee of standard modern predicate logic. Individuals and societies are thus taken to be able to "bootstrap" themselves up to higher levels of intellectual achievement.

The most primitive, heavily K-dependent basic-natural, logical system of quantification (basic-natural predicate logic or BNPL) was, in principle, codified by the Edinburgh philosopher Sir William Hamilton (1788-1856). Though looked down upon by the world of professional logicians, Hamilton's logic largely reflects what we find in natural language. This logic operates with the three mutually exclusive quantifiers ALL, SOME and NO. This means, inter alia, that the quantifier SOME entails NOT-ALL: at this level of logical analysis, when it is said that some flags are green, it is implied that not all flags are green - which is precisely what logically naive speakers take SOME to mean. This, however, goes against classical Aristotelian logic, where all flags are green entails that some flags are green.

The answer usually given is a bit strange. It is said, in Gricean pragmatics, that there is a principle tacitly accepted by interlocutors that one does not say more than one can vouch for (see below), so that, when one says some flags are green, one implicitly means that not all flags are green, because if all flags were green, one would have said that. This would then account for the clash with traditional Aristotelian logic, where the subaltern entailment from all to some holds. Yet in modern standard logic this entailment does not hold. So one naturally wonders what the Gricean pragmaticists are arguing for or against: they appear to be justifying natural intuitions in the face of Aristotelian logic, but at the same time they accept that Aristotelian logic is inadequate and has to be replaced by standard modern (Russellian) logic, where the entailment from all to some does not hold. So are the pragmaticists putting up a defence for a logical system that they themselves admit is inadequate? The truth seems to be that the pragmaticists themselves are unclear about the properties of the logical systems they try to defend natural language against.

The main drawback of basic-natural predicate logic is that it does not allow one to say that some flags are green until all flags have been checked, because if, in the end, it turns out that all flags are green, the sentence some flags are green will be false. This means that none of the three sentences all flags are green, no flags are green and some flags are green, can be vouched for until all flags have been checked. This is a serious

\footnotetext{
${ }^{1}$ Similar processes have been observed for numeracy, reading and geometrical competence (Dehaene 1997, 2005; Dehaene et al. 2006; Pica et al. 2004).
} 
epistemological drawback, inhibiting induction processes in cases where the totality of the quantification domain is too large, or lies partially in the future, so that it cannot be checked from beginning to end, as when we quantify over all humans or over all rainy days.

The more culturally developed form of predicate logic, embodied in the classic Square of Opposition - we call it the Square or strict-natural predicate logic (SNPL) is not this way dependent on our knowledge state $\mathbf{K}$, as it allows one to say that some flags are green even when not all flags have been checked, owing to its subaltern entailment from all to some: now some means 'some, perhaps all', so that we can say in truth some flags are green when of the unsurveyable mass of flags in the world at least some have been found to be green. This then allows one to extrapolate from these flags to all flags and venture the hypothesis that all flags are green, which will then quickly be shown to be false. But in some cases the hypothesis will hold and will not be falsified. In such cases we will add the hypothesis to what we consider to be our knowledge of the world, which will become more solid as the original hypothesis fails to be falsified. The Square and also standard modern predicate logic are thus helpful in the inductive process of constructing world knowledge in a way that BNPL is not. The transition from BNPL to the Square has thus been of immense importance to the human race: it is the transition from hic et nunc knowledge to generalized, and thus predictive, knowledge of the world.

The Square is the first $\mathbf{K}$-independent predicate logic in history. As a logical system, it is often falsely attributed to Aristotle (384-322 BCE), whose system of predicate logic is as defined in (14) below. The Square, as known in history, is the product of modifications to Aristotle's original logic, inserted by his commentators Apuleius $( \pm 125-180)$, Ammonius ( $\pm 440-520)$ and Boethius ( $\pm 480-524 / 5)$, who meant to streamline Aristotle's original system, thereby unwittingly introducing the logical error of undue existential import (UEI). This error leads to inconsistency when the set of entities quantified over is null. One of the reasons for the setting up of standard modern predicate logic (SMPL) by scholars like Frege, Peano, Russell was the necessity to remedy this fault. They eliminated UEI by basing logic firmly on standard Boolean set theory as developed from George Boole (1779-1848) onward. SMPL, however, violates natural logical intuitions to an unacceptable extent.

My thesis is that BNPL, which corresponds largely, but not entirely, with Hamilton's predicate logic, is the natural, $\mathbf{K}$-dependent logical system humans are born with. SNPL (the Square) reflects the natural logic resulting from freeing predicate logic from $\mathbf{K}$-dependence. Its defect of UEI is not remedied, in the natural logic of language, by the SMPL remedy, but by a distinction between two kinds of satisfaction conditions for predicates, the preconditions and the update conditions. The former preselect conditions for truth, with a view to discourse coherence, the latter pose final conditions for truth. Violation of the former gives radical falsity (F2); violation of the latter gives minimal falsity (F1). SMPL is all right for those domains where discourse plays no part. Natural 
logic takes discourse into account. Not recognising the discourse factor leads to empirical inadequacy of the logical system devised.

\section{The inadequacy of Gricean explanations}

The Gricean maxims are meant to account for the empirical clashes between intuitions and "standard logic", where it remains unclear, at least as regards predicate logic, whether by "standard logic" is meant the Square or SMPL (see above). The main difficulty with regard to "standard logic" revolves around the universal quantifier all or every for predicate logic (of either kind) and the conjunctive operator and for propositional logic. Natural intuition says that some excludes all, hence All $F$ is $G$ entails Not(Some $F$ is $G$ ). Yet the Square says that All $F$ is $G$ entails Some $F$ is $G$, whereas in SMPL the two are logically independent. Moreover, intuition says that not all is equivalent with some and that some is equivalent with some not. Likewise, and is felt to exclude or: $P$ and $Q$ is felt to entail $\operatorname{Not}(P$ or $Q$ ), but in standard propositional logic, $P$ and $Q$ entails $P$ or $Q$.

Yet instead of investigating the logical properties of systems where these intuitions hold, the Griceans posit a tacit understanding between speaker and hearer to the effect that one does not say more than one can vouch for and, by implication, that negation denies the minimum - an understanding based on the assumption of scales that range from minimum (weakest) to maximum (strongest) (Horn 1972). When John says Some flags are green, Dick will understand that John cannot vouch for the stronger statement All flags are green. Assuming that John has full relevant knowledge, the conclusion must be that it is not the case that all flags are green. Hence, Griceans say, there is an implicature, though not an entailment, from some to not all (the so-called only some reading) and vice versa from not all to some, and pragmatic equivalence of some and some not. Analogously, in propositional logic, there is an implicature from or to not and (the so-called exclusive or).

This may be true for some cases, but as a general explanation of the clash between natural logical intuitions and the theorems of either Aristotelian or standard modern logic, it must be deemed inadequate. First, it is based on the presumption of the speaker's full relevant knowledge, which is unsupported by any rational analysis of normal speech situations, where it is normal to take speaker's partiscience (nonomnicience) for granted (Hoeksema 1999: 5).

Then, there are discrepancies between natural logical intuitions and standard logic that are not accountable for in terms of Gricean maxims. For example, the maxims fail to account for the difference between the natural semantic processing of (1a) and (1b):

(1a) John doesn't like trains or planes (likes neither trains nor planes).

(1b) John doesn't like trains and planes. 
Sentence (1a), in either form, is immediately understood as 'John doesn't like trains and he doesn't like planes'. Yet (1b), with and instead of or, is not immediately understood as 'John doesn't like trains or he doesn't like planes'. One needs a considerable amount of sophisticated thinking to convince oneself that (1b) is equivalent with 'John doesn't like trains or he doesn't like planes'. This is remarkable, because, on the assumption that natural logic equals standard logic, standard propositional logic predicts that the same processing procedure should hold for the two, both being instances of De Morgan's laws of conversion between conjunction and disjunction.

The Gricean maxims have no bearing at all on such cases, but natural logic does. Basic-natural propositional logic has the three mutually contrary operators AND, (exclusive) OR and NEITHER...NOR (= German weder ...noch, French $n i . . . n i$, etc.) - corresponding to basic-natural ALL, SOME and NO, respectively, in predicate logic. At the level of logical intuitions, NEITHER P NOR Q is equivalent with NOT[P] AND NOT[Q], as in (1a). But NOT[P AND Q], as in (1b), is not equivalent, at that level, with NEITHER $P$ NOR $Q$ (though in standard propositional logic it is, due to De Morgan's Laws), but with EITHER [P OR Q] OR [NEITHER P NOR Q] (see Seuren 2010: 108-114).

Similar examples are drawn from the logic of relations, which has shown up a number of so-called paradoxes - cases where natural intuitions clash with apparently inviolate mathematico-logical analyses - all to do with reflexivity. Thus we have Russell's famous Barber Paradox: ${ }^{2}$

(2) Jones, who is a prisoner, shaves all the prisoners who do not shave themselves, and only those.

The question is: does or does not Jones shave himself? If he does, he does not and if he does not, he does. According to standard logic, Jones cannot exist, having the contradictory properties of both not shaving himself and not not shaving himself. Yet we, natural speakers, find this sentence perfectly understandable and we see no problem with it. Or take the sentence:

(3) All the girls in her class envy Trisha, who is the youngest girl of her class.

This sentence should entail that Trisha envies herself, but for natural speakers it in no way does. Or consider Peter Geach's well-known sentence pair (Geach 1962: 132):

(4a) Only Satan pities himself.

(4b) Only Satan pities Satan.

\footnotetext{
${ }^{2}$ Russell himself spoke not of a prison and prisoners but of a village and villagers, totally forgetting that a village normally contains women, who normally never shave themselves and would not be shaven by Russell's or any other barber. A prison is perhaps more acceptable from this point of view, as male and female inmates are still strictly separated.
} 
The question is: why do these two sentences not have the same meaning, as standard logical analysis seems to require? Or take the following little dialogue between a father and his young son who is crying because he has just hurt himself:

(5a) Father: Well-educated boys don't cry.

(5b) Son: Í didn't educate me!

If the boy had said:

\section{Í didn’t educate myself!}

his answer wouldn't have made sense and the effect would have been lost. Current theory will provide the following logico-semantic analysis of $(5 b)$ :

$$
\text { not [Educate }(\mathrm{I}, \mathrm{I})]
$$

with a discourse-driven contrastive accent overlay on the subject term $I$, considered to be of a pragmatic nature. But, given the grammatical process of reflexivising an object term that is co-referential with the subject term, (7) would give (6), not (5b), and (5b) will remain unexplained, both grammatically and semantically.

The Gricean maxims provide no satisfactory answer. Levinson (2000: 181, 277280), contrasting Only Felix voted for him with Only Felix voted for himself, posits a scale whereby a reflexive pronoun (himself) is more informative than a nonreflexive pronoun (him). The Gricean Maxim of Quantity ("make your contribution as informative as is required, not more") would then predict that the use of him "implicates" that the more informative himself is not intended by the speaker. The examples (2-5) show that this appeal to the Gricean maxims lacks generality and is thus ad hoc. It fails to apply to (2), which does not allow for the "less informative" them instead of themselves. It fails to apply to (3), which does not even contain a pronoun that could compete with a reflexive, and also to (4b), where the double occurrence of Satan imposes referential identity, just as the himself of (4a) - yet Levinson's principle fails to show up the semantic difference. Likewise for the difference between $(5 b)$ and (6).

In Seuren (1989) it is argued that these and similar cases are straightforwardly explained by the assumption of (a) a grammar that derives surface sentence structures from their underlying Semantic Analysis or SA, and (b) the True Binarity Principle, which applies to the logic of language and forbids codenotation of terms under the same predicate in SAs and imposes reflexivisation, which makes for a different but related reflexive predicate, when two (or more) clausemate terms do have the same denotation at SA-level. ${ }^{3}$

\footnotetext{
${ }^{3}$ I speak of denotation, not of referent, because what counts is not the real or actual world referent, if any, but the referent in the world as it would be if the particular world picture in which the proposition at hand is
} 
The requirement of a grammar that derives surface structures from underlying SAs is essential for the solution of cases like (4a,b) or (5b). (4a) and (4b) are then analysed, in informal terms, at SA-level as (8a) and (8b), respectively:

(8a) The class of those $x$ such that $x$ pities $x$ comprises Satan and nobody else.

(8b) The class of those $x$ such that $x$ pities Satan comprises Satan and nobody else.

Here, clausemate reflexivity ("x pities x") occurs only in (8a), not in (8b), so that (8a) requires reflexivisation whereas $(8 \mathrm{~b})$ does not.

Similarly for (5b) and (6), which are analysed at SA-level as (9a) and (9b), respectively:

(9a) Not [the $x$ such that $x$ educated me] is me.

(9b) Not [the $x$ such that $x$ educated $x]$ is me.

where clausemate reflexivity ("x educated x") occurs only in (9b), not in (a), so that $(9 b)$ requires reflexivisation whereas $(9 a)$ does not.

In general, given the inability of the Gricean maxims to bridge the gap between standard logic and natural intuitions, we try a different approach: we say that natural language has its own logic, which differs from standard logic. To see how this can work, we must first see what defines a logic.

\section{What is natural logic?}

Throughout history, logic has been defined in many different ways. One definition, which is in keeping with the tradition, is to say that logic is (the study of) the formally definable system responsible for consistency within a text (where "consistency" is taken in the sense of possible simultaneous truth). Some texts, such as mathematical texts, are themselves cast in a (largely) formal language requiring its own (mathematical) logic. We are speaking, however, of texts in some natural language, which, therefore, require what we call a natural logic for the maintenance of consistency. Natural logic is thus essential in the study of semantics: when we convey information, tell a story, issue an order or ask a question, we need to be consistent and logic is one way for us to fulfill that requirement.

As opposed to the rest of semantics, logic is formal by definition - that is, any logic is a calculus which, when followed, guarantees consistency for the sentences covered by it. For most forms of consistency, however, no calculus is available. Thus, we know

operative were a reflection of actual reality. Thus, if I think that John and the rapist are two different persons whereas they are, in fact, one and the same person, I can say, without any semantic anomaly, I think John will kill the rapist, not at all implying, thereby, that I think that John will kill himself. 
that (10a) is inconsistent with (10b) (with John referring to the same person), but there is no logical proof of it - unless lexical meaning descriptions are incorporated into the logic, as is proposed in Carnap (1956), or, in a totally different fashion, in Seuren (2010). Without any such machinery, all we can do is rely on our intuitive understanding of these sentences and then conclude, on mere intuitive grounds, that they are inconsistent with each other:

(10a) John teaches in Paris.

(10b) John died two years ago.

By contrast, the naturally felt inconsistency between (11a) and (11b) is supported by standard logic. All varieties of predicate logic tell us that (11b) entails the existence of at least one speaker of French, while (11a) blocks any such entailment:

(11a) Nobody speaks French.

(11b) Some speakers of French live in Paris.

For natural intuition, the following two are also inconsistent:

(12a) Nobody speaks French.

(12b) All speakers of French live in Paris.

But now logic does not speak with one mouth. In SMPL, (12a) and (12b) are taken to be consistent, because, in this logic, (12b) counts as true when there are no speakers of French. But in both BNPL and SNPL (the Square), which let all entail some, these two sentences are inconsistent with each other.

Aristotle (384-322 BCE) discovered that a formal theory of textual consistency crucially depends on a handful of words, called OPERATORS or CONSTANTS. For him as for us - these words are ALL, SOME, NOT, AND, OR, IF, MAY, MUST and a few more. In the present paper, we concentrate on ALL and SOME, and a little also on NOT. Apart from functional variables (usually $x, y, z$ ) that range over individuals, (first order) logical formulae thus consist of operators and variables, the latter ranging over propositions in propositional logic and over predicates in predicate logic. In any logic, the operators are defined as regards the conditions under which they produce truth (their satisfaction conditions). Standard logic uses satisfaction conditions that mirror standard settheoretic operations.

The empirical question now is: how are the operators - or rather, the words or morphemes that correspond to them in natural languages - defined in natural language (assuming that individual languages do not differ in this respect)? If we can define the natural-language logical operators in an empirically valid way, and if these definitions form a sound system of logic, we will have the natural logic of language and cognition we are trying to find (which is likely to differ in important ways from standard logic) 
and we will have eliminated the need for a pragmatic explanation for the clashes between natural logical intuitions and the great systems of logic known from history.

This argument seems incontrovertible. Yet it is not welcomed by professional logicians, who feel that their discipline is by definition not dependent on empirical data but is supported by mathematical, or even metaphysical, necessity and should not be relativised with regard to language in general or to particular languages. However, while it is correct to say that mathematical and metaphysical necessity constrain any logical system, as they constrain everything under the sun, the crucial point is that logic is supported by analytical - that is, semantic - necessity, and for semantic necessity one needs a language, and languages are empirical objects. The logic of language thus becomes a matter of lexical semantics. It is important that linguists should become aware of this fact.

\section{Four predicate logics}

Simplistically speaking, predicate logic is the theory of (universal and existential) quantification. Given the three quantifiers ALL, SOME and NO, and the negation NOT, either over the whole sentence/proposition (external negation) or over the predicate (internal negation), we distinguish the following twelve basic sentence types (without vacuous repetitions of negations; the variables $F$ and $G$ stand for predicates): ${ }^{4}$

\begin{tabular}{|c|c|c|c|}
\hline A & ALL $F$ is $G$ & $\neg \mathrm{A}$ & NOT [ALL $F$ is $G$ ] \\
\hline I & SOME $F$ is $G$ & 기 & NOT [SOME $F$ is G] \\
\hline $\mathbf{N}$ & NO $F$ is $G$ & $\neg \mathbf{N}$ & NOT [ $\mathrm{NO} F$ is $\mathrm{G}]$ \\
\hline$A^{*}$ & ALL $F$ is NOT-G & $\neg A^{*}$ & NOT [ALL $F$ is NOT-G] \\
\hline$\left.\right|^{*}$ & SOME $F$ is NOT-G & 기* & NOT [SOME F is NOT-G] \\
\hline $\mathbf{N}^{*}$ & NO $F$ is NOT-G & $\neg \mathbf{N}^{*}$ & NOT [NO F is NOT-G] \\
\hline
\end{tabular}

In those logics where NO equals NOT-SOME, the four $\mathbf{N}$-type sentences are otiose.

This notational system will do for the present purpose. But what do the words ALL, SOME, and NO mean? ${ }^{5}$ In order not to complicate matters too much right away, we take NOT to be the standard truth-value toggle in all four logics considered, even though, in

\footnotetext{
${ }^{4}$ These sentence types are defined for monadic sentence types (with only one quantifier, located in the subject term). Of course, language knows sentences with multiple quantification, such as All children know some football players. These are not discussed here for the simple reason that multiple quantification is always reducible to single quantification by means of so-called lambda abstraction: All children know some football players is reducible to 'All children are such that they [know some football players]', with 'know some football players' as predicate, which is again analysable as 'for $x$ to be such that there is at least one football player $y$ such that $x$ knows $y$ '. The actual logic is not affected by such analyses.

${ }^{5}$ For the sake of brevity, I will leave the semantic description of the logical connectives (NOT, AND, OR, IF) out of account. For a discussion of these, see Seuren $(2010$ : 50-52, 108-114).
} 
reality, natural language negation is far more complex than that. As regards ALL, SOME and NO, they are defined in SMPL as follows ( $\llbracket P \rrbracket$ stands for the extension of the predicate $\mathrm{P} ;<\mathrm{a}, \mathrm{b}>$ stands for the ordered pair 'a followed by b'):

(13) Standard Modern Predicate Logic SMPL:

$\llbracket A L L \rrbracket=\{<\llbracket F \rrbracket, \llbracket G \rrbracket>\mid \llbracket F \rrbracket \subseteq \llbracket G \rrbracket\}$

(the extension of the predicate ALL consists of the set of all pairs $\llbracket F \rrbracket$, $\llbracket \mathrm{G} \rrbracket$ such that $\llbracket F \rrbracket$ is included in or equals $\llbracket G \rrbracket$ )

$\llbracket \mathrm{SOME} \rrbracket=\{<\llbracket \mathrm{F} \rrbracket, \llbracket \mathrm{G} \rrbracket>\mid \llbracket \mathrm{F} \rrbracket \cap \llbracket \mathrm{G} \rrbracket \neq \varnothing\}$

(the extension of the predicate SOME consists of the set of all pairs $\llbracket F \rrbracket$, $\llbracket \mathrm{G} \rrbracket$ such that the intersection of $\llbracket F \rrbracket$ and $\llbracket \mathrm{G} \rrbracket$ is nonnull)

$\llbracket N O \rrbracket=\{<\llbracket F \rrbracket, \llbracket G \rrbracket>\mid \llbracket F \rrbracket \cap \llbracket G \rrbracket=\varnothing\}$

(the extension of the predicate NO consists of the set of all pairs $\llbracket F \rrbracket$, $\llbracket \mathrm{G} \rrbracket$ such that the intersection of $\llbracket F \rrbracket$ and $\llbracket G \rrbracket$ is null)

(Under these definitions, NO $F$ is $G$ is simply the negation of SOME $F$ is $G$.)

The relation with standard set theory is obvious. SOME simply requires nonnullness of the intersection of $\llbracket F \rrbracket$ and $\llbracket G \rrbracket$, whereas ALL requires inclusion (in the standard sense) of $\llbracket F \rrbracket$ in $\llbracket G \rrbracket$. For the rest, truth is determined by the laws and theorems of standard set theory. Thus, in SMPL, ALL F is $G$ is trivially true when $\llbracket F \rrbracket=\varnothing$, because in standard set theory the null set $(\varnothing)$ is a subset of any set. And SOME $F$ is $G$ is trivially true when both $\llbracket F \rrbracket$ and $\llbracket G \rrbracket$ are nonnull and $\llbracket F \rrbracket \supseteq \llbracket G \rrbracket$, because when these conditions are met, their intersection is nonnull.

It is well-known, however, that natural intuition does not support all such truth judgements. For example, ALL $F$ is $G$ is false for natural intuition when there are no $F_{s}$ and SOME $F$ is $G$ is felt to be false when $\llbracket F \rrbracket \subseteq \llbracket G \rrbracket$ (so that ALL $F$ is $G$ is true), but true when $\llbracket G \rrbracket \subset \llbracket F \rrbracket$. Aristotle, followed by Abelard (1079-1142), respected the former intuition but not the latter. Their logic, Aristotelian-Abelardian predicate logic or AAPL, is a perfectly sound alternative to SMPL, from which it differs only in that, in the absence of any Fs, ALL F is $G$ is considered false in the former but true in the latter. In AAPC, ALL is characterised by the following definition, while SOME and NO are as in (13):

(14) Aristotelian-Abelardian predicate logic (AAPL):

$\llbracket A L L \rrbracket=\{<\llbracket F \rrbracket, \llbracket G \rrbracket>\mid \llbracket F \rrbracket \subseteq \llbracket G \rrbracket$ and $\llbracket F \rrbracket \neq \varnothing\}$

(the extension of the predicate ALL consists of the set of all pairs $\llbracket F \rrbracket$, $\llbracket \mathrm{G} \rrbracket$ such that $\llbracket F \rrbracket$ is included in or equals $\llbracket \mathrm{G} \rrbracket$ and $\llbracket F \rrbracket$ is nonnull) 
$\llbracket \mathrm{SOME} \rrbracket=\{<\llbracket \mathrm{F} \rrbracket, \llbracket \mathrm{G} \rrbracket>\mid \llbracket \mathrm{F} \rrbracket \cap \llbracket \mathrm{G} \rrbracket \neq \varnothing\}$

(the extension of the predicate SOME consists of the set of all pairs $\llbracket F \rrbracket$, $G \rrbracket$ such that the intersection of $\llbracket F \rrbracket$ and $\llbracket G \rrbracket$ is nonnull)

$\llbracket N O \rrbracket=\{<\llbracket F \rrbracket, \llbracket G \rrbracket>\mid \llbracket F \rrbracket \cap \llbracket G \rrbracket=\varnothing\}$

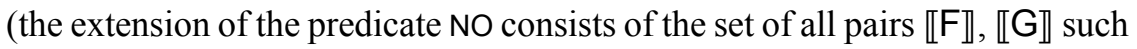
that the intersection of $\llbracket F \rrbracket$ and $\llbracket \mathrm{G} \rrbracket$ is null)

(Again, NO $F$ is $G$ is simply the negation of SOME $F$ is $G$.)

This formulation differs from (13) only in that there is the extra requirement for the truth of $\mathbf{A}$ (ALL $F$ is $G$ ) that $\llbracket F \rrbracket$ be nonnull.

But this fails to do justice to the other intuition that I (SOME $F$ is $G$ ) is considered false when either $\llbracket F \rrbracket \subset \llbracket G \rrbracket$ or $\llbracket F \rrbracket=\llbracket G \rrbracket$, but true when $\llbracket G \rrbracket \subset \llbracket F \rrbracket$ (which implies that $\llbracket G \rrbracket$ is nonnull). Our intuition tells us that SOME is to be read as 'some but not all' or 'only some'. To account for both intuitions, we define ALL, SOME and NO as follows:

(15) Basic-Natural Predicate Logic (BNPL):

$\llbracket A L L \rrbracket=\{<\llbracket F \rrbracket, \llbracket G \rrbracket>\mid \llbracket F \rrbracket \subseteq \llbracket G \rrbracket$ and $\llbracket F \rrbracket \neq \varnothing\}$

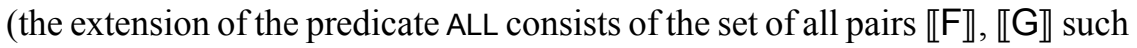
that $\llbracket F \rrbracket$ is included in or equals $\llbracket G \rrbracket$ and $\llbracket F \rrbracket$ is nonnull)

$\llbracket$ SOME $=\{<\llbracket F \rrbracket, \llbracket G \rrbracket>\mid \llbracket F \rrbracket \cap \llbracket G \rrbracket \neq \varnothing$ and $\llbracket F \rrbracket \cap \llbracket G \rrbracket \subset \llbracket F \rrbracket\}$

(the extension of the predicate SOME consists of the set of all pairs $\llbracket F \rrbracket$, $\llbracket \rrbracket$ such that the intersection of $\llbracket F \rrbracket$ and $\llbracket G \rrbracket$ is nonnull and is properly included in $\llbracket F \rrbracket)$

$\llbracket N O \rrbracket=\{<\llbracket F \rrbracket, \llbracket G \rrbracket>\mid \llbracket F \rrbracket \cap \llbracket G \rrbracket=\varnothing\}$

(the extension of the predicate NO consists of the set of all pairs $\llbracket F \rrbracket, \llbracket G \rrbracket$ such that the intersection of $\llbracket F \rrbracket$ and $\llbracket G \rrbracket$ is null)

(Now, NO $F$ is $G$ is not the negation of SOME $F$ is $G$ because NO $F$ is $G$ and SOME $F$ is $G$ are both false in cases where ALL $F$ is $G$ is true.)

This formulation differs from (14) only in that I (SOME $F$ is $G$ ) requires for truth not only that the intersection of $\llbracket F \rrbracket$ and $\llbracket G \rrbracket$ be nonnull but also that this intersection be properly included in $\llbracket F \rrbracket$, so that not all $F$ are $G$.

In the fourth predicate logic, the Square, the definitions of ALL, SOME and NO are as in (14), but for the fact that the commentators added the theorem of the so-called Conversions, which holds in SMPL but not in AAPC and even less in BNPC. The Conversions are defined as follows: 


$$
\left.\mathbf{A} \equiv \neg\right|^{*} \quad \text { and } \quad I \equiv \neg A^{*}
$$

That is, ALL and SOME are interchangeable provided an external and an internal negation are added. In current terminology it is said that ALL and SOME are duals in the systems concerned.

The Conversions do not follow from the semantic definitions of the quantifiers given in (14) but were added as independent elements, which is why they have led to the logical error of UEI: a sound logic is defined exclusively by the semantics of its operators; any further addition may (and perhaps will) make a logic unsound. ${ }^{6}$

Apart from the Square, which suffers from the logical defect of UEI, the other three systems are logically sound. The soundness of SMPL cannot be at issue. AAPL is as sound as SMPL (which should give standard logicians pause to think). BNPL as defined in (15) is logically sound, no matter whether situations where $\llbracket F \rrbracket=\varnothing$ are taken into or left out of account (see Seuren 2010: Ch. 3).

\section{Intuitions tested}

These four systems are now set off against the following seven natural logical intuitions ( $\vdash$ or 'entails', here: 'is felt to entail'; likewise for $\equiv$ or 'is equivalent', here: 'is felt to be equivalent'):

1. SOME $F$ is $G \vdash$ NOT [ALL $F$ is $G$ ]

2. SOME $F$ is $G \equiv$ SOME $F$ is NOT-G

3. SOME $F$ is $G \equiv$ SOME $G$ is $F$

4. ALL $F$ is $G \vdash$ SOME $G$ is $F$

5. ALL $F$ is $G \vdash$ SOME $G$ is NOT-F

6. NO $F$ is NOT-G $\equiv A L L F$ is $G$

7. NOT-ALL $F$ is $G \equiv$ SOME $F$ is $G \equiv$ SOME $F$ is NOT- $G$

$$
\begin{aligned}
& I \vdash \neg A \\
& I \equiv I^{*} \\
& I \equiv I !^{7} \\
& \mathbf{A} \vdash I ! \\
& \mathbf{A} \vdash I^{*} \\
& \mathbf{N}^{*} \equiv \mathbf{A} \\
& \neg \mathbf{A} \equiv \mathbf{I} \equiv \mathbf{I}^{*}
\end{aligned}
$$

The combination of 3 and 4 amounts to the positive subaltern entailment from ALL $F$ is $G$ to SOME F is G, found in AAPL and SNPL (the Square) but not in BNPL or SMPL. (As we have seen (note 2 above), the positive subaltern entailment schema is not fully or basically natural but emerges on further reflection.) The four systems score as follows:

\footnotetext{
${ }^{6}$ The Square can be salvaged by the addition of a presuppositional component to the logic, which then contains the Square as a proper subpart (see Seuren 1988, 2010: Ch. 10). This extension of the system also brings us a step closer to an empirically adequate analysis of the negation operator, which we have allowed to remain "classical" in the present paper.

${ }^{7}$ The exclamation mark stands for the so-called inversion of a sentence type. Thus, I! stands for 'SOME G is $F^{\prime}$.
} 
BNPC $1,2,4,5,6^{8}$

AAPC 3,4

ABPC 3, 4, 6

SMPL 3,6

BNPL scores best: it misses out only on the intuitions 3 and 7. AAPL and the SNPL (the Square) successfully account for the intuitions 3 and 4 . In both systems SOME is symmetrical (SOME $F$ is $G \equiv$ SOME $G$ is $F$ ) and ALL $F$ is $G \vdash$ SOME $G$ is $F$, because when $\llbracket F \rrbracket \subseteq \llbracket G \rrbracket$ and $\llbracket F \rrbracket \neq \varnothing, \llbracket F \rrbracket \cap \llbracket G \rrbracket \neq \varnothing$. SNPL has the extra advantage of accounting for intuition 6, since in that system NO F IS NOT-G and ALLF IS G are equivalent in virtue of the Conversions. Standard modern predicate logic (SMPL) accounts for the intuitions 3 (SOME is symmetrical) and 6 (NOF IS NOT-G $\equiv$ ALL F IS G in virtue of the Conversions).

None of the systems is able to account for intuition 7. The Square and SMPL come closest in that there NOT [ALL $F$ is $G$ ] $\equiv$ SOME $F$ is NOT-G, but SOME $F$ is $G$ and SOME $F$ is NOT-G are not equivalent in these systems. In BNPL, when NOT [ALL $F$ is G] is true, then either SOME $F$ is $G$ ( $\equiv$ SOME $F$ is NOT-G) or NO $F$ is $G$ is true. In AAPC, SOME $F$ is NOT-G - NOT [ALL $F$ is $G$ ] but not vice versa, because when $\llbracket F \rrbracket=\varnothing$, NOT [ALL $F$ is $G$ ] is true but SOME $F$ is NOT-G is false.

The explanation proposed falls back on topic-comment (TC) structure (information structure). TC structure is analysed as an underlying cleft where all has comment status, as in (17b). Thus, the analysis of a sentence like Ben didn't eat all of his meal, is 'what Ben ate of his meal was not all', entailing presuppositionally that Ben ate some of his meal and thus excluding the case that he ate nothing. Thus read, the sentence presupposes that Ben ate some of his meal and asserts that he did not eat all of it. Similarly for (17a), which, if analysed as (17b), excludes the case that there are no green flags:

(17a) Not all flags are green.

(17b) 'the flags that are green are not all (flags)'

Thus, to the extent that NOT-ALL denies the comment ALL, the intuitive equivalence of SOME $F$ is (NOT-)G with NOT-ALL F is $G$ is explained by topic-comment structure.

It seems that we have to conclude that the totality of natural logical intuitions held by logically naive humans does not fit into a single logical system. In order to account for all the intuitions, a distinction will have to be made between a basic-natural and a strict-natural system of predicate logic, the former being a close relative of Hamilton's predicate logic, the latter being equivalent to the classic Square of Opposition.

\footnotetext{
${ }^{8}$ The equivalence expressed in Intuition 6 follows from BNPL only when situations where $\llbracket F \rrbracket=\varnothing$ are left out of account, as they are by those who operate with BNPL as "their" predicate logic.
} 


\section{REFERENCES}

Carnap, R. 1956. Meaning and necessity. A study in semantics and modal logic. (Enlarged edition.) Chicago: The University of Chicago Press.

Dehaene, S. 1997. The number sense. New York: Oxford University Press.

Dehaene, S. 2005. "Evolution of human cortical circuits for reading and arithmetic: The "neuronal recycling' hypothesis". In: Dehaene, S., J.-R. Duhamel, M.D. Hauser and G. Rizzolatti (eds.), From monkey brain to human brain. Cambridge, MA: MIT Press. 133-157.

Dehaene, S., V. Izard, P. Pica and E. Spelke. 2006. "Core knowledge of geometry in an Amazonian indigene group". Science 311 (January 2006). 381-384.

Geach, P.T. 1962. Reference and generality. An examination of some medieval and modern theories. Ithaca, NY: Cornell University Press.

Hamilton, Sir William. 1866. Lectures on metaphysics and logic. (Vol. IV.) (=Lectures on logic. [Vol. II], 2nd ed., revised.) (Edited by H.L. Mansel and John Veitch.) Edinburgh and London: Blackwood \& Sons.

Hoeksema, J. 1999. "Blocking effects and polarity sensitivity”. In: Gerbrandy, J., M. Marx, M. de Rijke and Y. Venema (eds.), JFAK. Essays dedicated to Johan van Benthem on the occasion of his 50th birthday. Amsterdam: Amsterdam University Press. (Not available in hard copy). $<$ http://www.illc.uva.nl/j50/contribs/hoeksema/hoeksema.pdf $>$

Horn, L. 1972. On the semantic properties of logical operators in English. (Unpublished PhD dissertation, University of Southern Califiornia, Los Angeles.)

Levinson, S.C. 2000. Presumptive meanings. The theory of generalized conversational implicature. Cambridge, MA: MIT Press.

Pica, P., C. Lemer, V. Izard and S. Dehaene. 2004. "Exact and approximate arithmetic in an Amazonian indigene group". Science 306 (October 2004). 499-503.

Seuren, P.A.M. 1988. "Presupposition and negation". Journal of Semantics 6(3/4). 175-226.

Seuren, P.A.M. 1989. "Notes on reflexivity”. In: Heyvaert, F.J. and F. Steurs (eds.), Worlds behind words. Essays in honour of Prof. Dr. F.G. Droste on the occasion of his sixtieth birthday. Leuven: Leuven University Press. 85-95.

Seuren, P.A.M. 2006. "The natural logic of language and cognition". Pragmatics 16(1). 103138.

Seuren, P.A.M. 2010. The logic of language. (= Language from within [Vol. 2].). Oxford: Oxford University Press.

\section{Address correspondence to:}

Pieter A.M. Seuren

Max Planck Institute for Psycholinguistics

PO Box 310

6500 AH Nijmegen

The Netherlands

pieter.seuren@mpi.nl 